\title{
Evolución de la documentación en enfermería en España: las bases de datos Cuiden y Bdie
}

Por Alberto Gálvez Toro, Encarnación Poyatos Huertas y José Manuel Estrada Lorenzo

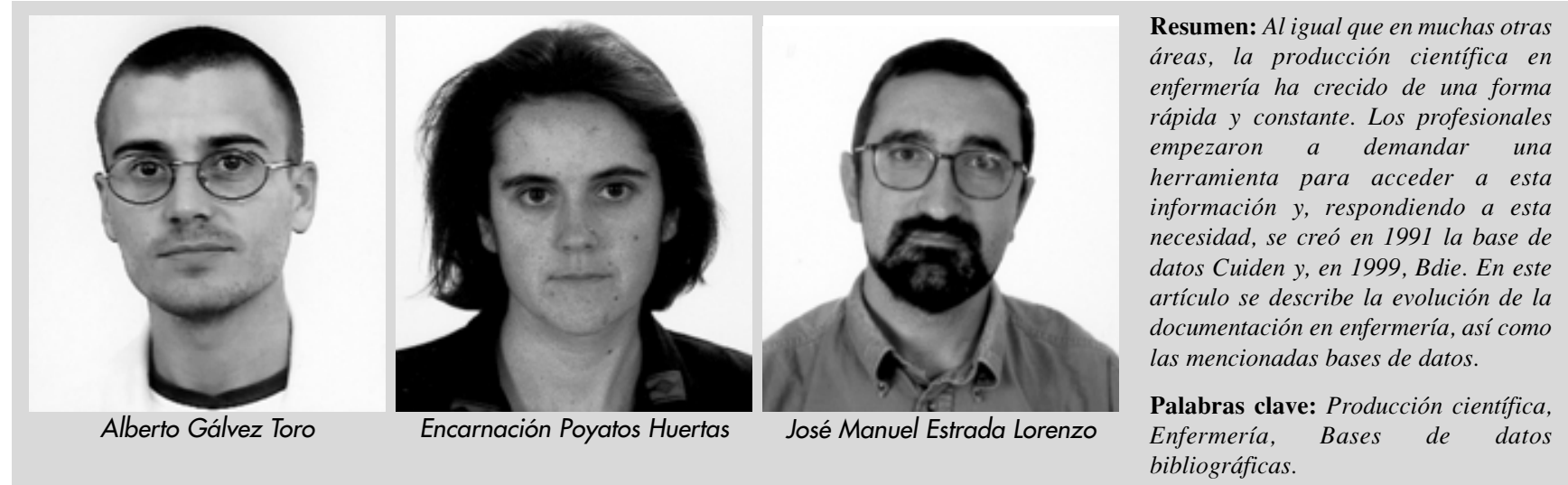

Title: Evolution of nursing documentation in Spain: the Cuiden and Bdie databases

Abstract: As has happened in many other fields, scientific output in the area of nursing has grown quickly and steadily. The health professionals demanded a tool to access this type of information and, in response, the Cuiden database was created in 1991 followed by the Bdie database in 1999. This article describes the development of nursing documentation, as well as the Cuiden and Bdie databases.

Keywords: Scientific output, Nursing, Bibliographic databases.

Gálvez Toro, Alberto; Poyatos Huertas, Encarnación; Estrada Lorenzo, José Manuel. "Evolución de la documentación en enfermería en España: las bases de datos Cuiden y Bdie". En: El profesional de la información, 2000, diciembre, v. 9, n. 12, pp. 13-19.

Con este trabajo se pretende dar a conocer entre la comunidad científica no especializada en enfermería la existencia de dos bases de datos bibliográficas complementarias, Cuiden y Bdie, dedicadas a la difusión de la producción científica española en enfermería. Tras un breve repaso a lo que ha sido el desarrollo de la documentación en este ámbito, se describen las principales características y funciones de dichas bases de datos.

\section{Nacimiento de la documentación científica en la enfermería española}

Si tomamos nota de las palabras de Castro Vizoso $^{1}$, uno de los factores implicados en el nacimiento de la enfermería profesional y científica en España fue el Decreto de 4 de diciembre de 1953 por el que se unifican las carreras sanitarias de enfermera, practicante y matrona con el nombre de asistente técnico sanitario (ATS). No obstante, los orígenes de la enfermería moderna en nuestro país, al menos tal y como la entendemos hoy, se podrían situar en 1977, cuando se publica el Decreto 2128/77 por el que las Escuelas de Asistentes Técnicos Sanitarios se transforman en Escuelas Universitarias de Enfermería ${ }^{2}$. No hay duda de que el lugar que hoy día ocupa la enfermería depende de lo ocurrido en el pasado (conferencia de Alma-Ata de 1978, Ley General de Sanidad de 1986, Real Decreto de Estructuras Básicas de Salud de 1984, etc.).

Sin embargo, de manera sinóptica, nos interesa resaltar lo que aportó la diplomatura de enfermería en el desarrollo documental. Fue el inicio de un proceso que dura hasta nuestros días y que ha supuesto, entre otros hechos cruciales, la formación de una comunidad docente estable y grupos de investigación consolidados distribuidos por toda España; el desarrollo de una investigación cada vez más rigurosa y adaptada a las necesidades de la enfermería y la evolución y proliferación de revistas científicas.

\section{«Salvo Cuiden, en el resto de las bases de datos conocidas la producción científica enfermera española no era una preocupa- ción primordial»}

No puede entenderse una disciplina universitaria que carezca de recursos bibliográficos y de publicaciones especializadas. Esta era la realidad de la enfermería española en 1977 ya que el antecedente histórico más cercano (ATS) había realizado pocas aportacio- 
nes a la literatura científica enfermera durante 25 años (la figura del ATS es una imagen distorsionada de la enfermera, que se crea como un profesional totalmente dependiente y subordinado al dictamen médico y que reproduce el patrón hombre/mujer y las diferencias de género). Además, las nuevas enfermeras miraban al antiguo profesional con recelo. Realmente se puede establecer una relación de causalidad directa entre el nacimiento de la enfermería universitaria (y el cambio de denominación de ATS a enfermera) y la generación de conocimiento nuevo (de enfermería, que no de $A T S$ ) y, por tanto, el aumento de la producción científica con el consiguiente aumento del consumo de información.

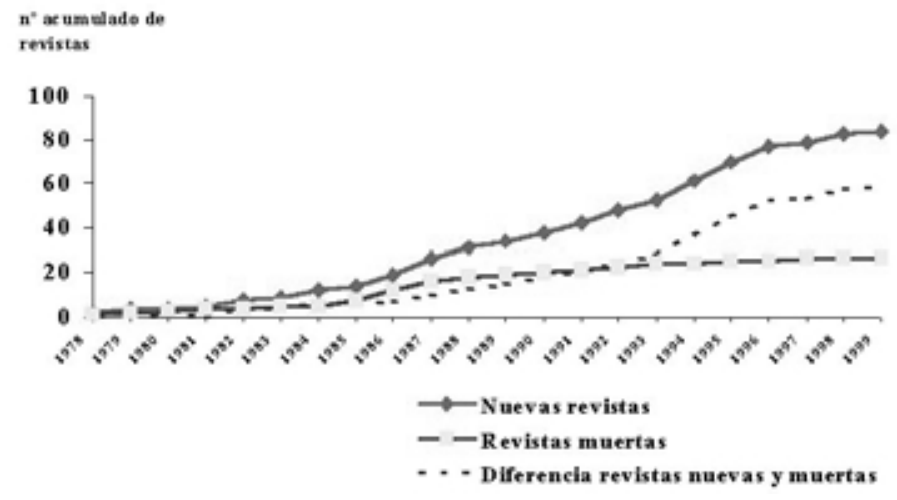

Figura 1. Crecimiento del número de publicaciones en enfermería (1978-2000). Fuente: Catálogo Rehic.

\section{Primera etapa: Elaboración de los primeros recursos documentales}

1977 -Decreto 2128/77. Enfermería accede a la Universidad.

1978 -Nace la primera revista de Enfermería. Escasos recursos bibliográficos. Traducción de textos del inglés: revistas, manuales, tratados.

1978-1990 -Progresivo aumento de la información enfermera española. Crecimiento del número de revistas circulantes.

Segunda etapa: Necesidad de facilitar el acceso a la documentación publicada.

1990 -Investigadores de enfermería recomiendan la creación de fuentes bibliográficas secundarias.

1991 -Base de datos bibliográfica Cuiden.

1992 -Revista bibliográfica Index de Enfermería (1992).

1993 -Base de datos bibliográfica Cuidatge.

1997 -Se celebra en Granada la 1르 Conferencia Nacional de Revistas de Enfermería y Afines. Consenso entre los editores de revistas de enfermería.

1999 -Base de datos bibliográfica Bdie.

Tercera etapa: Acceso universal a la producción científica de la enfermería española.

1998-1999 -Cuiden, Cuidatge y Bdie se pueden consultar gratuitamente en internet.

2000 -Desarrollo de los recursos documentales sobre enfermería en internet.

Tabla 1. Evolución de la documentación en enfermería en España

El vacío de conocimiento, muy notable en los primeros años, fue llenándose con la traducción de textos ingleses o norteamericanos (el ATS era una figura única y aislada, no tenía un homónimo conceptual, teórico o práctico al que acudir en el resto del mundo, pero las enfermeras españolas de 1977 sí que pudieron encontrar nurses, infirmière y enfermeras de las que aprender en otras partes). Se comenzaron a publicar re-

\begin{tabular}{|l|c|l|}
\hline \multicolumn{1}{|c|}{ Tipo de fuente } & $\begin{array}{c}\mathbf{N}^{\circ} \text { registros } \\
\text { bibliográficos }\end{array}$ & $\%$ \\
\hline Artículos & 16.481 & 86,7 \\
\hline Libros & 891 & 4,7 \\
\hline Capitulos & 624 & 3,3 \\
\hline $\begin{array}{l}\text { Ponencias y } \\
\text { comunicaciones }\end{array}$ & 501 & 2,6 \\
\hline Literatura gris & 454 & 2,4 \\
\hline Proyectos de investigación & 53 & 0,3 \\
\hline Material audiovisual & 8 & 0,04 \\
\hline Total & 19.012 & 100 \\
\hline
\end{tabular}

Tabla 2. Número y distribución de registros bibliográficos en Cuiden (mayo 2000) vistas extranjeras traducidas al castellano (como la edición española de Nursing, que aún hoy pervive) al tiempo que nacían las primeras publicaciones especializadas españolas.

El nuevo discurso, ATS frente a enfermera, comenzaba a tomar forma. En pocos años aumentó su número, entre las cuales las primeras en ver la luz fueron: Rol de enfermería (1978), Publicación científica para enfermería (1978), Nueva enfermería (1979), Revista internacional de enfermería (1981), Enfermería científica (1982) o Temas de enfermería (1982). Todos ellos vehículos de comunicación de esta incipiente comunidad científica necesarios para adoctrinar y dar contenido a la enfermería española (figura 1).

El proceso de aparición, desarrollo y desaparición de revistas se mantuvo durante las décadas de los años ochenta y noventa ${ }^{3,4}$. Hacia 1990 la enfermería española era víctima de la explosión de la información que otras ciencias ya experimentaban; la recuperación de datos, manual hasta entonces, era difícil e interminable; las hemerotecas de ciencias de la salud apenas sí 
tenían recursos documentales relativos a la disciplina y la mayoría de las colecciones estaban incompletas o habían terminado siendo destruidas $o$, con suerte, arrinconadas en un almacén. Empezaba a percibirse la necesidad de crear un instrumento que facilitase el acceso a la información de manera rápida y sencilla.

En 1991 se creó la base de datos bibliográfica Cuiden (Cuidados de enfermería) bajo los auspicios del Centro de Documentación de Enfermería Comunitaria ( $\mathrm{Cedec})$, más tarde Fundación Index (Granada), cumpliendo así uno de sus fines fundacionales: mejorar la difusión de la información científica especializada en cuidados de la salud, favoreciendo la accesibilidad de los profesionales e investigadores a las fuentes $\mathrm{y}$ fondos documentales ${ }^{5,6}$.

En 1993 apareció la base de datos bibliográfica Cuidatge, de la que es responsable la biblioteca de la Escuela de Enfermería de la Universidad Rovira $i$ Virgili, en Tarragona. Dispone de 15.000 registros y su temática es tanto enfermería como otras disciplinas afines. Contiene referencias de las publicaciones que se reciben en la biblioteca (artículos de revistas, folletos, etc.) siendo la mayoría de las revistas de ámbito estatal y con títulos especí-

\begin{tabular}{|l|c|c|c|c|}
\hline \multirow{2}{*}{\multicolumn{1}{|c|}{ Colecciones }} & \multicolumn{2}{|c|}{$\begin{array}{c}\text { Revistas } \\
\text { enfermería }\end{array}$} & \multicolumn{2}{c|}{$\begin{array}{c}\text { Otras revistas } \\
\text { ciencias salud }\end{array}$} \\
\cline { 2 - 5 } & n. & $\%$ & n. & $\%$ \\
\hline Revistas vivas & 66 & 68,0 & 39 & 88,6 \\
\hline Revistas muertas & 24 & 24,7 & 1 & 2,3 \\
\hline $\begin{array}{l}\text { No consta si están } \\
\text { vivas o muertas }\end{array}$ & 7 & 7,3 & 4 & 9,1 \\
\hline Total & 97 & 100,0 & 44 & 100 \\
\hline
\end{tabular}

Tabla 3. Revistas de la hemeroteca de Index

ficos de enfermería. Los datos que se introducen por cada registro bibliográfico son: autor, título (del artículo) y fuente (nombre de la revista, año, volumen y número), incluyéndose un campo de descriptores (materia en catalán).

Más recientemente, en 1999, empieza a funcionar la Base de datos para la investigación en enfermería en España (Bdie), fruto de la colaboración, entre otras instituciones, del Instituto de Salud Carlos III (Madrid) y la Fundación Index. A su vez, hay que reseñar otros dos esfuerzos dedicados a recopilar la documentación española sobre enfermería, como son los casos de $S a u ́ d e e^{7}$, base bibliográfica elaborada en la biblioteca del Hospital Juan Canalejo, y de Enfermundi ${ }^{8}$, de la que es responsable el Consejo General de Enfermería.

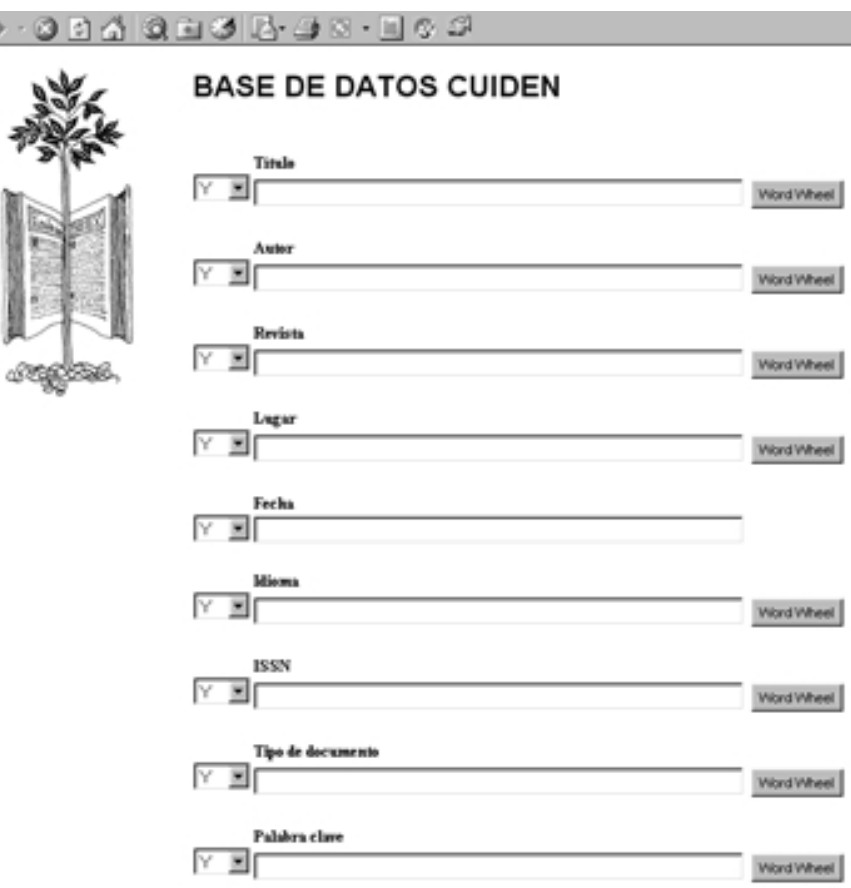

Figura 2. Parte de la pantalla de consulta bibliográfica de Cuiden

Desde 1977 puede decirse que la enfermería española ha pasado, en lo que respecta a la documentación científica, por tres etapas bien diferenciadas tras su incorporación a la universidad: búsqueda y creación de recursos bibliográficos, elaboración de bases de datos bibliográficas y universalización de los recursos documentales (tabla 1). Actualmente gracias a los instrumentos creados y al crecimiento de la producción científica, la investigación primaria y original está adquiriendo un peso importante en el conjunto de la información circulante y se están desarrollando líneas de trabajo sólidas basadas en fuentes propias ${ }^{9-17}$.

\section{La base de datos bibliográfica Cuiden}

Nace en 1991 con la finalidad de recoger de forma exhaustiva la producción científica de este campo en español. Actualmente cuenta con 19.012 registros (tabla 2) y está accesible desde internet (figura 2). Respecto a su cobertura temática, abarca las áreas de interés para la enfermería, incluyendo todas sus especialidades sin olvidar enfoques históricos, sociológicos o antropológicos del cuidado de la salud. Contiene registros bibliográficos de revistas de enfermería y áreas relacionadas (editadas en España e Iberoamérica y que

\begin{tabular}{|l|c|c|}
\hline \multicolumn{1}{|c|}{ Tipo de fuente } & $\begin{array}{c}\mathbf{N}^{*} \text { de registros } \\
\text { bibliográficos }\end{array}$ & $\%$ \\
\hline Proyectos investigación & 159 & 10 \\
\hline Tesinas & 303 & 20 \\
\hline Ponencias y comunicaciones & 10 & 1 \\
\hline Revistas de enfermería & 812 & 54 \\
\hline Revistas biomédicas & 198 & 13 \\
\hline Premios & 26 & 2 \\
\hline Total & 1508 & 100 \\
\hline
\end{tabular}

Tabla 4. Número y distribución de registros en Bdie (mayo 2000) 
AUTOR: Zabalegui Yarnoz, Adelaida

TÍTULO: Mecanismos de afrontamiento en cáncer avanzado, bajo tratamiento quimioterápico

REVISTA: Enferm Clínica

DESCRIPCIÓN FÍSICA: 1999 sep-oct; 9(5):185-193

INSTITUCIÓN: Facultad de Ciencias de la Salud. Universidad Internacional de Cataluña. Gomera, s/n.

08190 S. Cugat del Vallès

IDIOMA: Español

TIPO DE DOCUMENTO: Artículo original

ISSN: $1130-8621$

RESUMEN: Objetivo: Estudiar la relación entre los mecanismos de afrontamiento y la tensión psicológica en pacientes con cáncer avanzado que reciben tratamiento quimioterápico. Método: Estudio descriptivocorrelativo y análisis de regresión múltiple. Se usó como marco de referencia del estudio el modelo de 'stress and coping' de Lazarus y Folkman. Se estudiaron 132 pacientes con cáncer avanzado (mama, ovario, pulmón, colorrectal, etc.) de edades comprendidas entre 33 y 83 años, y procedentes de hospitales públicos o privados de Nueva York. Los mecanismos de afrontamiento de huida cognitiva, comportamiento de escape, distanciamiento, enfoque positivo y búsqueda de apoyo social se midieron con el instrumento Ways of Coping Inventory, Cancer Version (WCI-CV). La tensión psicológica se midió con el Profile of Mood States (POMS). Resultados: El distanciamiento estaba negativamente relacionado $(r=-0,25)$, mientras que la huida cognitiva $(r=0,38)$ y el comportamiento de escape $(r=0,38)$ estaban relacionados positivamente con la tensión psicológica $(\mathrm{p}<0,01)$. Colectivamente, los mecanismos de afrontamiento supusieron el 36\% de la variancia de la tensión psicológica. Los mecanismos de afrontamiento más importantes en cuanto a su contribución a la tensión psicológica fueron el comportamiento de escape (beta $=0,40$, $\mathrm{p}<0,001$ ), seguido de la huida cognitiva (beta $=0,29, \mathrm{p}<0,001$ ), y del distanciamiento (beta $=-0,24, \mathrm{p}<$ $0,01)$. Discusión: Los grados bajos de tensión psicológica estaban asociados con grados bajos de huida cognitiva y de comportamiento de escape, y con grados elevados de distanciamiento

PALABRAS CLAVE: Cáncer; Enfermedades; Oncología; Quimioterapia

Figura 3. Modelo de registro bibliográfico Cuiden

la Fundación Index recibe mediante intercambio), monografías, capítulos de libros, ponencias y comunicaciones a congresos, literatura gris, proyectos de investigación y material audiovisual (figura 3 ).

\section{http://www.index-f.com}

Durante años, la única forma de acceder a la base de datos Cuiden fue mediante el Servicio de Información Bibliográfica (SIB) que podían utilizar los suscriptores de la revista Index de enfermería y también las instituciones con las que la Fundación Index mantenía un convenio de colaboración. A través de este servicio se podían solicitar búsquedas bibliográficas en la base de datos que se realizaban en la sede de di-

\section{BDIE}
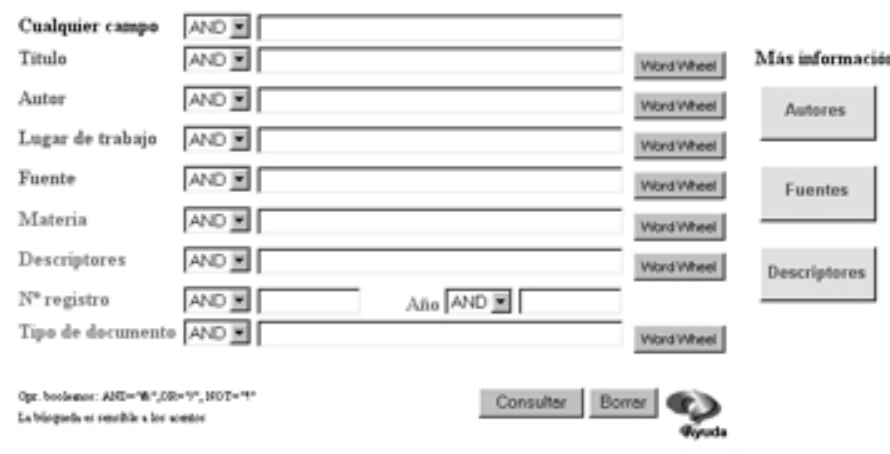

Figura 4. Pantalla de consulta bibliográfica Bdie cha fundación y, a continuación, se enviaban a los interesados por correo, fax o correo electrónico. Esta situación ha cambiado en la actualidad gracias a la colaboración entre la empresa de documentación Doc6 e Index, lo que ha permitido su acceso a través de internet de forma libre y gratuita.

Otra de las funciones de Cuiden es la de servir de apoyo a la revista Index de enfermería, ya que a partir de la primera esta publicación edita dos índices complementarios: uno de autores, con sus correspondientes referencias bibliográficas y otro de materias. Además, la Fundación Index ha desarrollado otras dos bases de datos (de momento no disponibles en internet):

-Rheic (Revistas de la hemeroteca de Index indizadas en Cuiden) (tabla 3). Es un catálogo electrónico de las publicaciones periódicas presentes en la colección de la Fundación Index. Cumple una doble función: aporta información sobre las características de las publicaciones periódicas de enfermería y muestra al usuario los fondos documentales de dicho centro ${ }^{3}$.

- Bdlic (Base de datos del laboratorio de investigación cualitativa). Incluye registros de documentos en español sobre investigación cualitativa o que utilizan en su desarrollo la metodología cualitativa. Cuenta actualmente con 1.102 registros. 


\section{Bdie, una propuesta complementaria}

Se origina a partir de un proyecto de investigación financiado por el Fondo de Investigación Sanitaria (FIS) en 1998, en el que están colaborando en la actualidad la Unidad de Investigación en Servicios de Salud y la Biblioteca Nacional de Ciencias de la Salud, ambas pertenecientes al Instituto de Salud Carlos III (Isciii) en Madrid y la Fundación Index en Grana$\mathrm{da}^{18}$ (figura 4).

\section{http://www.isciii.es/investen/bdie}

Previamente a la solicitud del proyecto de investigación se había analizado la situación de la documentación sobre enfermería en España ${ }^{19}$. En primer lugar se habían detectado las necesidades del personal especializado para actualizar sus conocimientos, derivadas del nuevo rumbo que había tomado la disciplina en los últimos años, donde la teoría y la investigación han ido adquiriendo un papel más relevante. Sin embargo se tropezaba con la difícil accesibilidad al conocimiento enfermero y con la escasa difusión de la producción científica nacional. Puede decirse que, salvo Cuiden, en el resto de las bases de datos conocidas la producción científica sobre enfermería no era una preocupación primordial (por ejemplo, el Índice médico espa- ñol con problemas de actualización y más volcado en el conocimiento médico; Medline o Cinahl donde, por su naturaleza, la producción científica enfermera española representa un pequeñísimo porcentaje) $)^{20-22}$.

\section{«Intencionadamente se ha pre- tendido que Bdie no fuera sólo una mera base de datos biblio- gráfica»}

Finalmente Index e Isciii llegaron a un compromiso de colaboración del que surgió, tras la confirmación de la financiación por el FIS, la base de datos Bdie. Dos son sus principales propósitos: cubrir las facetas de la producción científica que Cuiden sólo abarca de forma parcial (por ejemplo, proyectos de investigación, trabajos de postgrado de alumnos de enfermería y literatura gris) y hacerla accesible de forma gratuita y universal a través de su correspondiente página web en internet. Bdie se encuentra a disposición de los usuarios desde noviembre de 1999.

Su núcleo principal lo constituye la información bibliográfica, recopilada en formato de registros (figura 5). Un registro recoge los campos habituales de una

№: 1998.054

TITULO: Enfermería basada en la evidencia (EBE): un nuevo desafío profesional

AUTOR: Icart Isern MT

LUGAR DE TRABAJO: Departamento de Enfermería de Salud Pública, Salud Mental y Maternoinfantil. Escuela Universitaria de Enfermería. Universidad de Barcelona (L'Hospitalet de Llobregat, Barcelona)

FUENTE: Enferm Clin

COLACIÓN: 8(2): 77-83

AÑO: 1998

TIPO DE DOCUMENTO: Artículo

ISSN: $1130-8621$

NOTA: Contiene 55 ref. y 5 tab.

MATERIA Investigación en enfermería

DESCRIPTORES: Medicina basada en la evidencia / Enfermería / Práctica profesional / Investigación en enfermería

RESUMEN El desarrollo de una práctica asistencial basada en la mejor evidencia científica disponible es un deseo compartido por los proveedores y los clientes/usuarios de todo sistema sanitario. Se espera que la enfermera, como proveedora de cuidados, sea capaz de identificar e investigar sobre los problemas reales y potenciales que afectan la salud de los clientes/usuarios y que actúe desde su ámbito (asistencial, docente o gestor) basándose en resultados derivados de estudios fiables y válidos. Pero la aplicación de estos resultados es un proceso complejo que exige la integración de la experiencia clínica personal y de la evidencia obtenida a través de revisiones sistemáticas. Precisamente de la práctica enfermera basada en la evidencia científica trata la enfermería basada en la evidencia (EBE). La EBE es un proceso en el que los problemas se presentan como preguntas cuya respuesta se busca y evalúa sistemáticamente a partir de los resultados de la investigación más reciente y que sirve de base para la toma de decisiones. Este artículo analiza los antecedentes de la EBE, define sus elementos conceptuales, comenta las necesidades que justifican su aplicación, explica sus etapas y valora sus limitaciones y perspectivas.

CENTRO DE REFERENCIA: C-HJC / GR-FI / M-BNCS 
base de datos bibliográfica, haciendo hincapié en los destinados a la materia (de carácter genérico) y descriptores (de tres tipos: de contenido, identificadores y topónimos). La información recogida en campos como autores, título, fuente y colación se ajusta a las normas de Vancouver, de uso extendido en el ámbito de las ciencias de la salud. Por su parte, los descriptores han sido seleccionados en su mayoría del tesauro DeCS (Descriptores en ciencias de la salud), realizado por Bireme, que es una traducción de las palabras clave del MeSH (Medical subject headings).

Intencionadamente se ha pretendido que Bdie no fuera sólo una mera base de datos bibliográfica y por ello al núcleo bibliográfico principal hay que añadir además:

1. Información sobre los componentes y las instituciones que configuran el grupo Bdie.

2. Información detallada sobre la metodología seguida por el grupo Bdie en la elaboración e indización de los registros bibliográficos (se incluye también información precisa sobre las fuentes que han sido indizadas hasta la actualidad y el número total de registros que corresponde a cada uno de los tipos de fuente) (tabla 4).

3. Bases de datos complementarias sobre autores, fuentes y palabras clave, que aportan al usuario una información adicional a la proporcionada por el registro bibliográfico (figura 4).

4. Interrelación con los usuarios. Mediante un buzón de correo electrónico se permite al usuario contactar con el grupo Bdie y dirigir directamente sus críticas, comentarios y sugerencias.

\section{bdie@isciii.es}

5. Enlaces a otras direcciones de interés, con especial énfasis en otras bases de datos especializadas, como Cuiden, Cuidatge o Pubmed, que permiten al usua-

\section{Versión online de EPI}

Existe una versión electrónica de la revista $E I$ profesional de la información, de uso gratuito para la mayoría de los suscriptores (empresas, organismos, instituciones), consultable en:

http://www.swetsnet.nl/

Más información en:

http://www.swets.nl/sps/journals/jonline.html rio obtener una visión más completa de la producción científica enfermera.

\section{Cuiden y Bdie, a modo de análisis comparado}

Teniendo la precaución de advertir que Cuiden lleva ya 10 años de experiencia y que Bdie apenas ha cumplido 1 año dando servicio - lo que implica ya unas diferencias claras en cuanto a volumen de información y capacidad de difusión - no es arriesgado señalar que ambas cumplen una función de complementariedad, en cierta medida explicada porque en las dos interviene, en distinta proporción, el centro de documentación de la Fundación Index.

\section{«Es posible que Cuiden y Bdie, consideradas en su conjunto, abarquen un alto porcentaje de la producción científica publica- da en castellano relativa a esta disciplina»}

Cuiden se ha esforzado desde sus orígenes en recopilar la producción científica de las publicaciones especializadas en enfermería, de las cuales indiza más de 40 y, en los últimos años, está dedicando una especial atención a la información editada en revistas del ámbito hispanoamericano. Por su parte, Bdie, sin olvidar la indización de las principales revistas (como Enfermería clínica, ROL, Index, Enfermería integral o Metas), está más preocupada por la recopilación de proyectos de investigación en este campo evaluados por agencias financiadoras españolas y de tesinas y trabajos presentados en escuelas de salud pública y escuelas de enfermería. Por ello, a no muy largo plazo, es posible que Cuiden y Bdie, consideradas en su conjunto, abarquen un alto porcentaje de la producción científica publicada en castellano relativa a esta disciplina.

Sin ánimo de profundizar demasiado en esta presentación sí puede decirse que ambas ofrecen ciertas semejanzas en cuanto a la estructura de sus registros bibliográficos: campos básicos como título, autor y fuente, además de la filiación de los autores y resumen (siempre y cuando aparezca en el documento). El tratamiento documental parte de la utilización común de una herramienta básica como es el tesauro DeCS, elaborado por Bireme, para el control de las palabras clave y la indización de contenidos. Sin embargo, esta herramienta está siendo explotada con dos filosofías distintas: mientras que $B d i e$ ha optado por una mayor restricción en el vocabulario (de ahí la existencia de una base de datos complementaria, descriptores, donde se señala a los usuarios los términos aceptados y los no 
aceptados), Cuiden ofrece una mayor libertad en su uso. Esta divergencia en el modo de gestionar el vocabulario controlado de la indización es otra muestra más de la complementariedad existente entre ambas.

Por último, en lo que respecta a las interfaces de consulta (figuras 2 y 4), las dos bases de datos utilizan un mismo software de recuperación de la información, recurren a la combinación de los operadores booleanos elementales $($ and $=\&$, or $=/$ y not=!) y permiten la posibilidad de realizar búsquedas bibliográficas de forma sencilla (en cualquier campo) o avanzada (eligiendo algunos específicos). Cuiden, además, ofrece la ventaja adicional de poder seleccionar los documentos una vez ejecutada la búsqueda.

Dado que Bdie todavía está en construcción no puede hacerse un análisis comparado más exhaustivo, si bien puede decirse que en estos momentos son más complementarias que excluyentes, dados sus diferentes criterios de selección de fuentes y documentos para indizar y la distinta filosofía de indización así como el empleo de los descriptores. En un futuro, un análisis bibliométrico más detallado y una evaluación de usuarios permitirá conocer si es válida su existencia complementaria al servicio de la comunidad científica enfermera. Sí parece evidente, en definitiva, que puedan entenderse como un fiel reflejo de lo que se ha investigado y publicado sobre la disciplina en España en los últimos años.

\section{Notas}

1. Castro Vizoso, J. Identidad de la enfermería como profesión. Granada: Colegio de Enfermería, 1993.

2. Ventosa Esquinaldo, F. Historia de la enfermería española. Madrid: Ciencia, 1984.

3. Gálvez Toro, A.; Poyatos Huertas, E. "Catálogo Rehic de publicaciones periódicas de la Fundación Index". En: Index de enfermería, 1999, v. VIII, n. 27, pp. 54-56.

4. Poyatos Huertas, E. "Cuiden: una base de datos para los profesionales del cuidado de la salud”. En: Index de enfermería, 1996, n. 16-17, pp. 85-86.

5. Grupo de trabajo de enfermería. "La investigación en enfermería en España”. En: Medicina clínica, 1994, v. 102, pp. 584-588.

6. Gálvez Toro, A.; Poyatos Huertas, E. "Index: 10 años de documentación enfermera en España. Aportaciones de la primera revista especializada en bibliografía española". En: Index de enfermería, 1998, v. VIII, n. 2021, pp. 11-15.

7. Blanco Pérez, A.; González Guitián, C. "Saúde, base de datos del Hospital Juan Canalejo, un producto propio". En: VI Jornadas de información y documentación en ciencias de la salud, 1996, pp. 203-208.

8. "La enfermería crea su propia base internacional de datos". En: Inform salud, 1996, n. 9, pp. 450-453.

9. Icart, M. T.; Pedreny, R.; López, R. et al. "La investigación en enfermería a través de los artículos publicados en las revistas ROL (1982-90) y
Enfermería científica (1984-90)". En: Enfermería científica, 1991, n. 116, pp. 33-41.

10. Martínez Vizcaíno, V.; Martínez Orozco, M. A. "Investigación de enfermería en atención primaria de salud: publicaciones de los años 198990”. En: Enfermería científica, 1992, n. 119, pp. 18-20.

11. Cabrero, J.; Richart, M. "Análisis de la literatura empírica de la investigación enfermera española”. En: Enfermería científica, 1992, n. 122, pp. 37-39.

12. Martínez, V.; Martínez, A. "Investigación de enfermería en atención primaria de salud: publicaciones de los años 1990-1991”. En: Enfermería científica, 1993, v. III, n. 2, pp. 54-57.

13. Torra Bou, J. E. "Producción científica de la enfermería española: un análisis a través de los artículos publicados en tres revistas durante el trienio 1991-1993”. En: ROL, 1995, n. 198, pp. 61-71.

14. Gálvez Toro, A. Producción científica de la enfermería española: evaluación de los años 1993-1994. En: Index de enfermería, 1996, n. 16-17, pp. 17-21.

15. Torra I Bou, J. E. "Estudio bibliométrico de los artículos publicados en tres revistas españolas durante los años 1991 al 1993". En: ROL, 1996, n. 218 , pp. 33-40.

16. González Soriano, M.; Risco Otaolaurruchi, C.; Freixas Pascual, M. J. "Evolución de las publicaciones de salud pública y enfermería comunitaria en series revistas españolas durante 10 años”. En: Enfermería clínica, 1999 , v. 7 , n. 5, pp. 217-225.

17. Blas Robledo, M.; Blaya López, N.; Casado Blanco, E.; Durán Duque, M.; Herrán Cardeñoso, S.; Lapuente Jambrina, G. et al. "Estudio bibliométrico de las publicaciones de matronas durante el período 19801995”. En: Matronas hoy, 1999, n. 12, pp. 15-24.

18. Estrada, J. M.; Blanco, A.; Mariño, L.; Poyatos, E.; Lázaro, P.; Grupo Bdie. "El acceso a la producción científica en enfermería: la base de datos bibliográfica Bdie". En: I Congreso nacional de informática y enfermería, 1999.

19. Estrada, J. M.; Blanco, A.; Llano, J.; Poyatos, E.; Lázaro, P.; Moreno, M.; Grupo Bdie. "Acceso automatizado a la bibliografía sobre enfermería en España”. En: III Congreso nacional de informática de la salud, 1999.

20. Estrada, J. M.; Blanco, A.; Llano, J.; Poyatos, E.; Moreno, T.; Grupo Bdie. "Difusión de la producción científica de la enfermería española en bases de datos bibliográficas". En: VIII Jornadas de información y documentación en ciencias de la salud, 1999.

21. Coma Campmany, I.; Montcusí Puig, C. "Fuentes bibliográficas para artículos de revista: base de datos Cinahl”. En: Enfermería clínica, 1997, v. 7 , n. 1 , pp. $39-41$.

22. Coma Campmany, I.; Dios Sánchez, R.; Montcusí Puig, C. "Fuentes de información para enfermería: comparación entre las bases de datos Cinahl y Medline”. En: Metas enf, 1999, n. 14, pp. 21-27.

Alberto Gálvez Toro y Encarnación Poyatos Huertas. Fundación Index (Granada). José Manuel Estrada Lorenzo. Instituto de Salud Carlos III (Madrid).

agtoro@teleline.es

indexcd@interbook.net

jestrada@isciii.es 